\title{
Could Excluded Patients Make a Change to the Research Results? Comment on "Music does not Alter Anxiety in Patients with Suspected Lung Cancer Undergoing Bronchoscopy: A Randomised Controlled Trial"- European Clinical Respiratory Journal
}

\author{
Elisabeth Jeppesen ${ }^{*}$ and Klaus R Larsen
}

Department of Respiratory Medicine, Bispebjerg Hospital, Copenhagen, Denmark

"Corresponding author: Elisabeth Jeppesen, Department of Respiratory Medicine, Bispebjerg Hospital, Copenhagen, Denmark, Tel: +4538635266; E-mail: Elisabeth.Margrethe.Langvall.Jeppesen@regionh.dk

Received date: February 03, 2017; Accepted date: February 28, 2017; Published date: March 02, 2017

Copyright: ( $) 2017$ Jeppesen E, et al. This is an open-access article distributed under the terms of the Creative Commons Attribution License, which permits unrestricted use, distribution, and reproduction in any medium, provided the original author and source are credited.

\section{Commentary}

The aim of the study was to investigate whether MusiCure could relieve pre-procedural anxiety for patients with suspected lung cancer undergoing bronchoscopy. "MusiCure-music as medicine" is music composed by Niels Eje and composed to act calming and soothing [1].

We found, at first, with simple analysis a significant difference between the music and the control group on anxiety measured by Spielberger's State-Trait Anxiety Inventory, but when adjusting for sex and baseline anxiety there was no significant difference.

Patients included in our study had a median anxiety score of 39 (20) at admission. One might wonder whether the anxiety score would be higher if the patients who declined participation were counted in.

In our study there were 419 patients assessed for eligibility, of these 79 declined participation, for different reasons, such as not tolerating earplugs, disliking the music available, worrying about not getting music if participating, but also quite a few declined participation because of worrying too much about the procedure to have the energy to participate, having to answer questionnaires about how they felt.

\begin{tabular}{|l|l|l|}
\hline Numerical code & Frequency & Percent \\
\hline 0 & 69 & 26.6 \\
\hline 1 & 34 & 13.1 \\
\hline 2 & 17 & 6.6 \\
\hline 3 & 10 & 3.9 \\
\hline 4 & 7 & 2.7 \\
\hline 5 & 79 & 30.5 \\
\hline 6 & 16 & 6.2 \\
\hline 7 & 17 & 6.6 \\
\hline 8 & 5 & 1.9 \\
\hline 9 & 5 & 1.9 \\
\hline Total & 259 & 100.0 \\
\hline Reason & & \\
\hline
\end{tabular}

Reason for exclusion: $0=$ impaired hearing, $1=$ not understanding Danish, $2=$ impaired memory, $3+9=$ organizational , $4=$ sedation with Propofol, $5=$ do not wish to participate, $6=$ not able to write by themselves, $7=$ Transthoracic needle biopsy, 8=Esophageal ultrasound

Table 1: Frequency of reason for exclusion.

170 patients did not meet the inclusion criteria as having impaired hearing or memory, not understanding Danish, not being able to write themselves, having other invasive investigations combined with bronchoscopy, or being sedated with propofol instead of midazolam and fentanyl (Table 1).

It is interesting to look at the excluded patients, because of the potential of making a change to the research results. The excluded patients in our study were significantly older and sicker, having significantly lower BMI and lower lung function. There was no significant difference regarding sex (Table 2). We found sex to be a major confounder in our study. The fact that women had a significantly higher anxiety score than men is not a new finding, so either women are more anxious than men or more willing to admit it [2]. The distribution between the sexes was the same with relation to the included and the excluded patients. This could hardly screw the results in our study.

\begin{tabular}{|l|l|l|l|}
\hline & Included & Excluded & P \\
\hline Female/Male & $73 / 78$ & $127 / 132$ & 0.9 \\
\hline Age $^{\wedge}$ & $63.7(11.6)$ & $69(16)$ & $0.006^{*}$ \\
\hline $\begin{array}{l}\text { FEV1 percent of } \\
\text { predicted a }\end{array}$ & $75.0(22.3)$ & $68(22)$ & $0.002^{*}$ \\
\hline FEV1/FVC ratio ^ & $68.4(12.1)$ & $69(20)$ & 0.2 \\
\hline BMI a & $25.4(5.4)$ & $23.8(4)$ & $0.008^{*}$ \\
\hline $\begin{array}{l}\text { a Data are expressed as mean (SD) } \\
\text { ^ Data are expressed as median (interquartile range, IQR) }\end{array}$ & \\
\hline
\end{tabular}

Table 2: Included patients vs excluded patients.

That the excluded patients were sicker than the included with lower lung function is not known to have affected the result. Earlier studies with music and bronchoscopy though do not report lung function [3-5].

The excluded patients were significantly older in our study. Age could possibly influence the music taste, and it is in general a weakness in our study that researcher-selected music was used instead of 
Citation: Jeppesen E, Larsen KR (2017) Could Excluded Patients Make a Change to the Research Results? Comment on "Music does not Alter Anxiety in Patients with Suspected Lung Cancer Undergoing Bronchoscopy: A Randomised Controlled Trial"- European Clinical Respiratory Journal. J Aging Sci 5: 172. doi:10.4172/2329-8847.1000172

Page 2 of 2

patient-selected music [6,7]. There is not much research on elderly people's music preference and few taking into account age when analysing the effect of music on anxiety. El-Hassan et al. divides patients by the age of 51 when randomised. Their reason for doing so is based on their observation that older patients have a better tolerance to upper and lower gastrointestinal endoscopic procedures. They found a significant difference between the control group and the music group on STAI-State regardless of age, but in their study patients selected music by themselves [8]. One study reports that elderly people prefer popular music of their young adult years and in their study there are no significant differences in preferences for sedative or stimulative music [9]. This study is though not in a hospital setting and does not take into account what elderly persons prefer to listen to when sick. Our conclusion is that age could possibly influence results when researcher selected music is used.

In our study we excluded patients who did not understand Danish due to the questionnaires. Cultural differences are known to influence music taste. In a mini-review about cultural differences in music chosen for pain relief Caucasians tended to choose orchesteral music and African-Americans tended to choose jazz as their first choice. MusiCure is orchestral music. If the excluded immigrants were included the assumption could be that the music chosen would fit worse for their musical taste.

In our sample, even though we did not find MusiCure to alter anxiety for patients with suspected lung cancer undergoing bronchoscopy, we found a significant difference in how patients rated their experience of the sound in the operating theatre, the music group finding it significantly more pleasant than the control group. MusiCure might therefore possibly enhance patients' comfort in the operating theatre in a Caucasian setting [10].

\section{References}

1. http://musicure.com/

2. Spielberger CD, Gorsuch RL, Lushene RE (1970) State-Trait Anxiety Inventory. Paolo Alto, CA: Consulting Psychologists Press.

3. Dubois J, Bartter T, Pratter MR (1997) Music improves patient comfort level during outpatient bronchoscopy. Journal of Bronchology \& Interventional Pulmonology 4: 83.

4. Colt HG, Powers A, Shanks TG (1999) Effect of music on state anxiety scores in patients undergoing fiberoptic bronchoscopy. Chest Journal 116: 819-824.

5. Triller N, Erzen D, Duh S, Petrinec Primozic M, Kosnik M (2006) Music during bronchoscopic examination: the physiological effects: A randomized trial. Respiration 73: 95-99.

6. Gillen E, Biley F, Allen D (2008) Effects of music listening on adult patients' preprocedural state anxiety in hospital. Int J Evid Based Healthc 6: 24-49.

7. Bradt J, Dileo C, Shim M (2013) Music interventions for preoperative anxiety. Cochrane Database Syst Rev 6: CD006908.

8. El-Hassan H, Mckeown K, Muller AF (2009) Clinical trial: music reduces anxiety levels in patients attending for endoscopy. Aliment Pharmacol Ther 30: 718-724.

9. Gibbons AC (1977) Popular Music Preferences of Elderly People. J Music Ther 14: 180-189.

10. Good M, Picot BL, Salem SG, Chin CC, Picot SF, et al. (2000) Cultural Differences in Music Chosen for Pain Relief. J Holistic Nursing 18: 245-260. 